\title{
Physical and Numerical Modelling of the Flow Structure behind Structure in Tsunami-like Flow
}

\author{
Hidenori Ishii, Waseda University, wj1d2d3b@akane.waseda.jp \\ Tomoya Shibayama, Wasedal University, shibayama@waseda.jp \\ Jacob Stolles, University of Ottawa, jstol065@uottawa.ca
}

\section{Introduction}

Coastal structures are expected to play a role as evacuation buildings. Numerous studies have been examined tsunami-induced wave force acting on an inland building (Shigihara et al., 2009; Arimitsu et al., 2014). In addition, coastal structures have the potential to obstruct inundating tsunami waves, protecting structure further from the coastline. Hydraulic experiments and numerical calculations on shielding effect are being carried out. Alternatively, the vortices from these structures can result in significant scour downstream, influencing downstream structure stability. In the 2011 Tohoku Region Pacific Offshore Earthquake, many cases were reported that the structure was scrubbed and the structure fallen down. Skakiyama et al. (2007) and Sakakiyma et al. (2008) pointed out that the standing vortex generated around the structure winds sand and causes scouring. Therefore, understanding the vortices generated around the structure is also important in predicting the scouring phenomenon. As such, the flow properties around coastal structures must be considered and acknowledged within the design process.

However, there are a few studies focusing on the flow fields behind structure.Arnason et al. (2009) measured velocity fields of dam-break flow around/in the wake of a vertical column. Wei et al. (2015) reproduced the experiment performed using $\mathrm{SPH}$. However, in the experiment, vortexes are not considered and the influence of the existence of structures on the surroundings has not been sufficiently considered. Therefore, in this study, the flow fields of a tsunami-like wave around structures were examined through hydraulic experiments. This experiment was performed to clarify the local vortex structure behind structures by measuring the plane flow velocity field. Also, the effect of obstructing protecting the land side structure is assessed by measuring the wave force behind structures. Furthermore, a three-dimensional model (OpenFOAM) was used to further analyze the flow, focusing on water-level and wave forces behind the structure.

\section{Hydraulic experiment}

A tsunami wave basin with a width of $4 \mathrm{~m}$, a length of $9 \mathrm{~m}$ and a depth of $0.5 \mathrm{~m}$ was used as shown in Figure 1. The basin was separated into a still-water (sea) and dry (land) section. Still-water depth was maintained at $0.2 \mathrm{~m}$. On the North side of the basin, a wave maker was installed whose operation is based on releasing a volume of water with a defined head with respect to the still water depth of the basin. After initializing the release of the water, the reservoirs emptied under the influence of gravity. A flow characterized by a solitary wave front profile was generated. A structure with a plan area of $0.10 \mathrm{~m} \times 0.10$ $\mathrm{m}$ was installed on land section. A load cell was installed behind the structure to measure load behind the structure as well as wave gauges (WG) to measure water depths. Surface particle image velocimetry (PIV) was performed to measure the surface flow velocity field. Using PIV, the flat flow field downstream of the structure could be discerned. In order to clarify the vortex structure behind the structure, the mean flow velocity was subtracted from the flow field in the tsunami progression. Figure 2 shows stream lines after the mean flow velocity was subtracted from the vector field. Large symmetrical separating vortex pairs were generated behind the structure and transported downstream within the primary flow field. The vortices were not observed to alternate on the left and right, like Karman vortex street, instead occurring simultaneously on both sides of the structure. It was also found that formation of the region, where flowing flows merged, influences the size and location of the vortex behind the structure.

\section{Numerical simulation}

For numerical calculation, interFoam, which is a two-phase flow solver of uncompressed / immiscible fluid in OpenFOAM was used. The solver adopts a finite volume method as discretization method and Volume Of Fluid method (VOF) for the free-surface fluid analysis model. Large Eddy Simulation was used for the turbulent model. The flow condition observed by experiment was reproduced well by the model. However, the water level and wave force behind the structure was somewhat overestimated. The cause of the overestimation is likely interfacial blur due to calculation error of the VOF method. Figure 4 shows some samples of streamlines in front of the structure in 3D-view and side view. The flow reaches the front of the structure and turn toward the bottom. Then the flow near the center line runs toward upstream about $10 \mathrm{~cm}$. Eventually it runs toward downstream along the sidewall of the structure. This streamline in front of the structure indicates that there is a turbulent eddy formed by the wave-structure interaction.

\section{Conclusion}

In this study, the structure of separation vortices formed behind structures was observed. Additionally, the flow condition behind the structure was reproduced through the numerical model, OpenFOAM. The flow velocity field was roughly in agreement with the experimental results, though the water level and wave force were overestimated by the numerical model. The authors believe that improvement of the free-surface capture method is necessary to improve calculation accuracy.

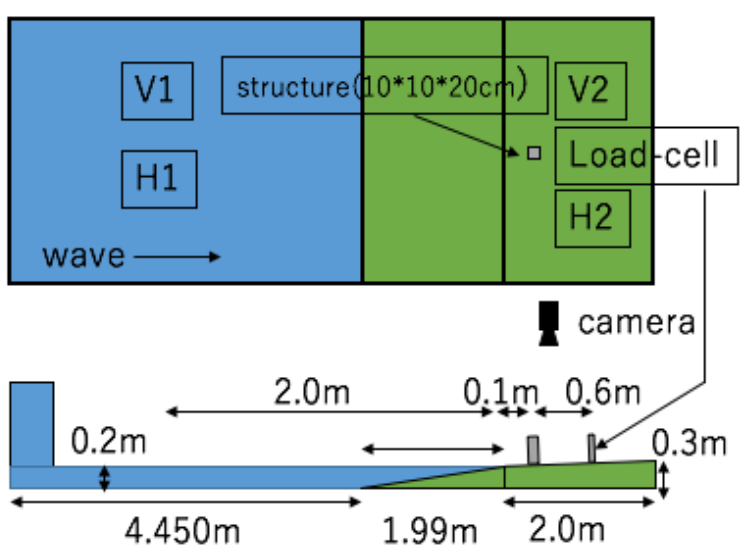

Figure 1 - Experiment setup 


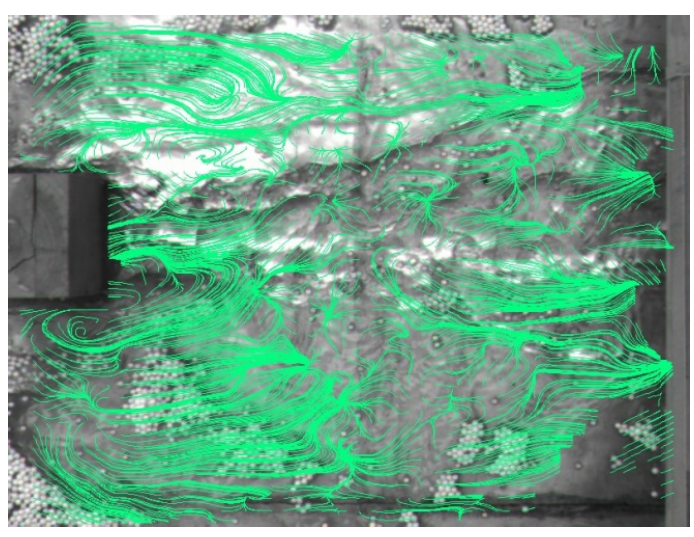

Figure 2 - Stream lines of flow vector obtained by subtracting average flow velocity in wave traveling direction

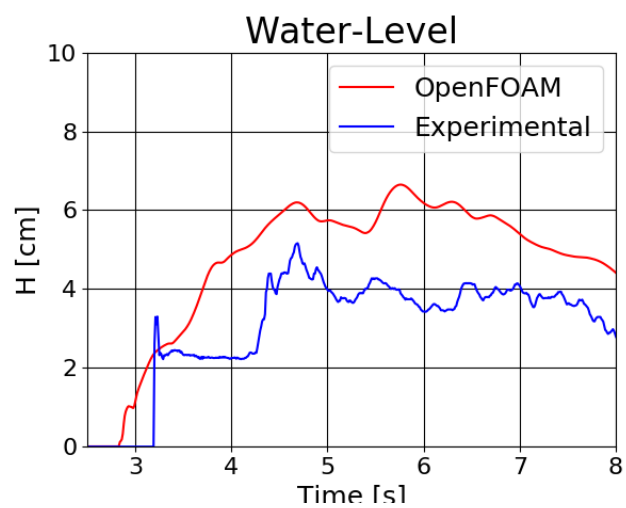

Figure 3 - Time-history of water-level

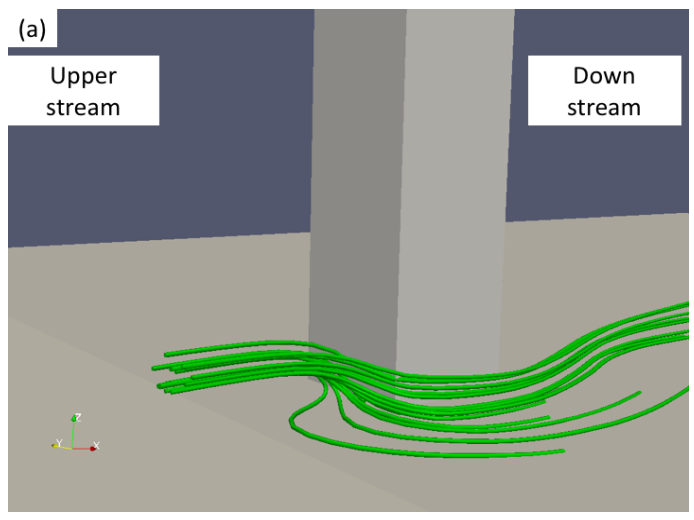

(b)

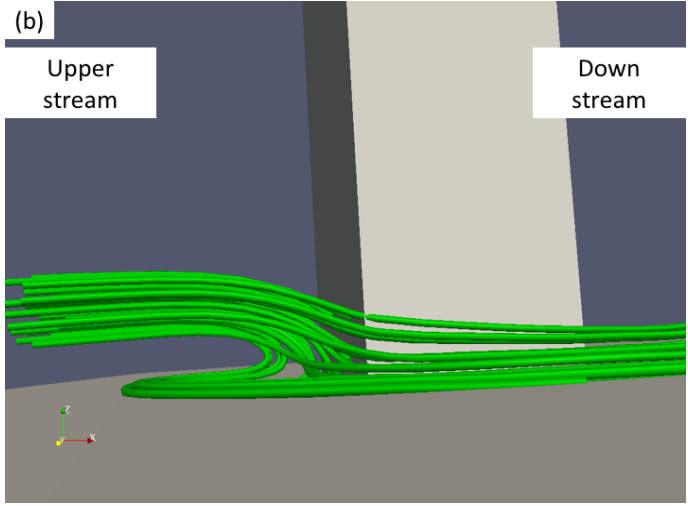

Figure 4 - samples of stream lines. (a) 3-D view, (b) side view

\section{REFERENCES}

Y. Shigihara, H. Iwase, K. Fujima and Y. Kotake, 2009. Evaluation of tsunami wave force by practical technique - Numerical simulation of wave force to act on standing structures, Journal of JSCE(A1), Vol. 65,No.1, P 905-913.

T. Arimitsu, H. Ono, K. Ooe, T. Deguchi and K. Kawasaki 2014. Applicability of evaluation method of tsunami wave force using $2 \mathrm{D}$ depth-integrated flow simulation results under installation of land structure, Journal of JSCE (B2), Vol. 70,No.2,P 786-790

T. Sakakiyama, M. Matsuyama, N. Kihara, 2007. Experimental Study on Bottom Topography Change in Harbor due to Tsunami, Proceedings of Coastal Engineering, JSCE, Vol. 54, P 506-510

T. Sakakiyama, M. Matsuyama, N. Kihara, 2008. Effects of tsunami velocity field on bottom topography change in harbor, Proceedings of Coastal Engineering, JSCE,Vol. 55,P 256-260.

Arnason, H., Petroff, C., Yeh, H., 2009. Tsunami Bore Impingement onto a Vertical Column. J. Disaster Res. 4, 391-403.

Wei, Z., Dalrymple, R.A., Hérault, A., et al., 2015. SPH modeling of dynamic impact of tsunami bore on bridge piers. Coast. Eng. 104, 26-42. 